(Stiasny-Kolster K et al. Neurology December (2 of 2) 2004;63:2272-2279). Augmentation or worsening of symptoms, unique to dopaminergics, occurs less often with cabergoline than with shorter acting drugs ( $9 \% \mathrm{cf} 30 \%$ with pramipexole). Cabergoline has a half-life of up to 65 hours, and is currently approved for treatment of adults with Parkinsonism. Pramipexole is currently considered the first line of treatment for idiopathic RLS (Silber M et al. Mayo Clinic Proc 2004;79:916-922).

\title{
CLINICAL FEATURES OF IDIOPATHIC PAROXYSMAL KINESIGENIC DYSKINESIA
}

The clinical features of 121 patients, referred for genetic study, with a presumptive diagnosis of idiopathic paroxysmal kinesigenic dyskinesia (PKD), were reviewed by a multicenter panel and reported from the University of Calfornia, San Francisco. Patients were interviewed by a single neurologist. Based on the distinctive homogeneous phenotype elicited in $79 \%$ of affected subjects, the authors propose the following diagnostic criteria for idiopathic PKD: attacks, mainly dystonic, triggered by sudden movements (100\%); precipitated by anxiety or stress $(62 \%)$, or caffeine $(13 \%)$; a premonitory sensation $(82 \%)$; short duration of attacks ( $<1$ minute; $<30 \mathrm{sec}$ in $93 \%)$; no loss of consciousness; painless; response to antiepileptic drugs (carbamazepine or phenytoin); no recognized alternative organic disease and normal neurologic exam; and age at onset between 1 and 20 years. Cases were familial or sporadic. Familial kindreds had a more variable age at onset, and a frequent history of infantile convulsions. The infantile-onset group of PKD cases $(n=12)$ had less uniform characteristics: a kinesigenic trigger was often absent, attacks sometimes occurred during sleep, and the response to AED (carbamazepine) was not universal. Sporadic cases were more often male, the attack frequency was higher, and males had a lower remission rate than females. Women had a better prognosis and a higher rate of complete remission; many obtained remission during pregnancy. (Bruno MK, Hallett M, Gwinn-Hardy $\mathrm{K}$, et al. Clinical evaluation of idiopathic paroxysmal kinesigenic dyskinesia. New diagnostic criteria. Neurology December (2 of 2) 2004;63:2280-2287). (Reprints: Dr LJ Ptacek, Howard Hughes Medical Institute, Department of Neurology, Universiy of California, San Francisco, $15504^{\text {th }}$ St, Mission Bay 19B, Rm 546, San Francisco, CA 94143).

COMMENT. This large clinical study of both familial and sporadic cases of PKD provides diagnostic criteria for idiopathic PKD. The age at onset is crucial ( $<20$ years), except in familial cases, and secondary causes such as multiple sclerosis, and vascular, metabolic, or traumatic disorders need to be excluded in patients presenting after 20 years of age. The presence of familial cases is not essential, but a family history of PKD makes the diagnosis more certain. A small group of infantile-onset cases do not conform to strict diagnostic criteria of $\mathrm{PKD}$, and alternative diagnoses include paroxysmal nonkinesigenic dyskinesia (PNKD), occurring without a trigger, shuddering attacks, and benign myoclonus of early infancy. Gene discovery in the future should help to clarify the diagnosis in doubtful cases.

Dystonia in a boy with secondary progressive multiple sclerosis is reported from Kyoto, Japan. (Shiraishi K et al. Brain Dev Dec 2004;26:539-541). Presenting at 8 years of age, the dystonia and MS were at first relapsing-remitting, and at 13 years, became progressive. 